Applied Physics Report 94-13

COND-MAT/9404077

\title{
Soft Disorder Effects in the Conductance Quantization in Quantum Point Contacts: Indirect Backscattering Statistics
}

\author{
Alexandre M. Zagoskin*, Sergey N. Rashkeev ${ }^{\dagger}$, Robert I. Shekhter ${ }^{\ddagger}$, and Göran Wendin ${ }^{\S}$ \\ Department of Applied Physics, Chalmers University of Technology and University of \\ Göteborg, S-41296 Göteborg, Sweden
}

\begin{abstract}
The breakdown of conductance quantization in a quantum point contact in the presence of random long-range impurity potential is discussed. It is shown that in the linear response regime a decisive role is played by the indirect backscattering mechanism via quasilocalized states at the Fermi level; this can provide much higher backscattering rate than any direct backscattering process. For the realistic contact lengths $(\leq 2000 \mathrm{~nm})$ the scattering processes prove to be independent, in spite of coherence of the electron wave. The distribution function of conductance fluctuations is obtained by direct numerical calculations as well as estimated within an analytical model for the first time. It is shown to be a generalized Poisson distribution. Estimates are made for quantum point contact performance at different choice of parameters. In particular, it is the better the larger the
\end{abstract}

\footnotetext{
*Email:alexz@fy.chalmers.se

†Email:sergey@fy.chalmers.se

${ }^{\ddagger}$ Email:shekhter@fy.chalmers.se

§mail:wendin@fy.chalmers.se
} 
intermode distance is compared to the amplitude of the random impurity potential.

PACS: 72.10.Bg, 72.20.Dp, 73.20.Dx 


\section{INTRODUCTION}

The effect of conductance $2 e^{2} / h$-quantization in quantum point contacts (QPC, electrostatically defined junction in a two-dimensional electron gas in a high mobility GaAsAlGaAs heterostructure), first observed in 1988 [1], is still challenging both theoreticians

ant experimentalists [2]. An intriguing feature of the effect is that it is not destroyed by elastic scattering, be it impurity scattering or scattering by a contact boundary.

The insensitivity of quantization to the latter process was explained within the framework of the adiabatic approximation [3]. Breakdown of quantization was shown to be insignificant as long as the contact shape (i.e., the confining potential induced by the gate electrode) is smooth on the scale of Fermi wavelength, $\lambda_{F}$. The last condition is likely to be true, since $\lambda_{F} \simeq 40 \mathrm{~nm}$ in a clean contact is at least an order of magnitude less than the characteristic scale of the gate potential variation [4].

The adiabatic approximation yields that different transverse modes pass the QPC independently ("no-mode-mixing" regime). This provides a sufficient, but not necessary, condition for the conductance quantization in a QPC: as was pointed in 田.5], the necessary condition is the absence of backscattering. The direct numerical calculations [6] show that conductance is quantized even if the intermode mixing is significant. The sum rule suggested there to explain this result follows from the unitarity of the scattering matrix of the system [7].

The impurity potential is more likely to produce backscattering, and thus to break the conductance quantization, since it is less smooth than the gate-induced potential. Many theoretical papers dealing with impurity scattering in QPC [8,9] used the Anderson model with on-site disorder. This approach gives a qualitative understanding of the process, but its results cannot be directly applied to real GaAs planar structures, where the Coulomb impurity potential certainly is not sharp on the scale of $\lambda_{F}$, 10. In fact, the numerical calculations made for this realistic case [4] show that its scale of variation is intermediate between $\lambda_{F}$ and the size of the QPC itself.

The effect of such a slowly varying random potential ("soft disorder") on the per- 
formance of a QPC was investigated in [4, 10]. Its main feature is the negligible rate of scattering processes with large momentum transfer. Glazman and Jonson [10] built a theory of the electrical conductivity through the QPC with soft disorder and showed that the direct backscattering occurs mainly in the largest-number transverse mode (i.e., one with the minimal longitudinal momentum). On another hand, Laughton et al. [4] demonstrated that the long range impurity potentials may produce quasilocalized states inside the channel [11]. Once appeared, such a state provides an effective indirect backscattering mechanism. Indeed, the quasilocalized state contains both "forward" and "backward" waves, so that the transition between the electronic states propagating in opposite directions via the quasilocalized state does not demand a significant momentum transfer. Thus, in an appropriate impurity configuration, indirect backscattering (formally due to the second order process) can have much larger cross section than the (first order) direct backscattering. The probability to find such a configuration of impurities, of course, must be investigated as well. Recently Gurvitz and Levinson [12] built a theory of resonant reflection and transmission in a QPC with a single attracting impurity at the contact's bottleneck. They have investigated the case when the first mode is open and a second one is being opened, thus creating a single resonant level at the top of the potential well. They obtained a Breit-Wigner type expression for the correction to the conductance at the transition region from the first to second conductance step (as function of the Fermi energy); its sign depends on whether there takes place tunneling into the resonant state (conductance enhancement) or scattering into it (suppression).

In this paper we study numerically and analytically the indirect backscattering effects on the electrical conductivity of a QPC, in the presence of the screened Coulomb potential from randomly distributed charged impurities.

In Sec [I] we find the corresponding correction to the current, supposing that there are quasilocalized states in the QPC, and that the impurity potential is soft, so that the tunneling processes to and from these states can be neglected, and their energetic spectrum is dense. We show that the correction, given by a Breit-Wigner type formula, suppresses the current. If the quasilocalized states exist close to the Fermi surface, this is 
really the leading contribution from the impurity scattering in a QPC. The result is valid for the bulk of the conductance step vs. gate voltage.

In Sec.III we investigate the stochastic properties of arising conductance fluctuations, as a random variable determined by the specific impurity arrangement. The distribution function of the conductance fluctuations is shown to be of Poisson type and is explicitly determined by the tunable parameters of the QPC: its length and number of open modes (i.e., conductance). This allows us to make more detailed predictions about the QPC performance than mere knowledge of the fluctuation dispersion.

In Sec.[V] we estimate the theory parameters based on the quasiclassical description of the random impurity potential.

In Sec. $\mathrm{V}$ we present the numerical calculations that provide the basis for the present work. The impurity potential was calculated in a self-consistent way using the ThomasFermi approximation. The quantum contact was modelled by imposing upon it a parabolic confining potential. The conductance was calculated in the linear responce limit by a standard transfer matrix method for different contact lengths and for different realizations of the impurity potential. ( Different realizations of the random impurity potential in the QPC was performed by changing the position of the confining potential with respect to once obtained random potential profile.)

An empirical distribution function of conductance fluctuations was thus obtained, and we compare the results of the numerical calculations and theoretical predictions in Sec.VI. They are in very good agreement. This allows us to conclude that the leading mechanism of conductance quantization breakdown in QPCs is the indirect backscattering, and it allows us to find estimates of the QPC performance.

\section{INDIRECT BACKSCATTERING IN A QUANTUM POINT CONTACT}

In accordance with what is now the standard approach [3, 10], we start from the adiabatic model of a QPC, where the transverse modes are well defined; the deviations will be regarded as a perturbation (see Fig.11). The Hamiltonian of the electron contains the 
following terms:

$$
H=-\frac{\hbar^{2}}{2 m^{*}}\left(\frac{\partial^{2}}{\partial x^{2}}+\frac{\partial^{2}}{\partial y^{2}}\right)+U_{g}(x, y)+V(x, y)
$$

Here $U_{g}(x, y)$ is the confinement potential, shaping the junction in a two-dimensional electron gas (2DEG). It proves to be convenient to include the smooth component of the impurity potential as well. The rest of impurity potential, $V(x, y)$, is regarded as a perturbation, e.g., leading to intermode mixing. It is also a soft potential, which does not lead to large momentum transfer.

The potential $U_{g}(x, y)$ is supposed to be a slow function of the coordinates. Then the wave function of an electron with energy $E$ inside the QPC can be expanded as follows [3]:

$$
\Psi(x, y ; E)=\sum_{m, \alpha} C_{m, \alpha} \psi_{m, \alpha}(x, y ; E)
$$

The adiabatic eigenfunctions are defined by

$$
\psi_{m, \alpha}(x, y ; E)=\phi_{m}(x, y) \chi_{m, \alpha}(x ; E)
$$

where the transverse eigenfunction corresponds to the $m$-th eigenvalue of the transverse Hamiltonian:

$$
H_{\perp} \phi_{m}(x, y) \equiv\left\{-\frac{\hbar^{2}}{2 m^{*}} \frac{\partial^{2}}{\partial y^{2}}+U_{g}(x, y)\right\} \phi_{m}(x, y)=E_{m \perp}(x) \phi_{m}(x, y)
$$

and the longitudinal eigenfunction satisfies the equation

$$
\left\{-\frac{\hbar^{2}}{2 m^{*}} \frac{\partial^{2}}{\partial x^{2}}+E_{m \perp}(x)\right\} \chi_{m, \alpha}(x ; E)=E \chi_{m, \alpha}(x ; E) .
$$

We can distinguish different groups of electronic states in the QPC, according to the behaviour of $\chi_{m, \alpha}(x ; E)$ in the presence of the effective one-dimensional potential $E_{m \perp}(x)$ (see Fig.尹). They are denoted by the index $\alpha=-2,-1, . .2$.

The states of special interest for us are the quasilocalized ones $(\alpha=0)$. They appear if the effective potential $E_{m \perp}(x)$ is a nonmonotonous function. Note that for each quasilocalized state in $m$-th mode, generally, there exist propagating states with the same 
energy in some mode of less number (Fig.2). This is the reason why we these states are quasilocalized (due to smoothness of the potentials in the QPC we can safely neglect another reason for this, namely, the tunneling decay).

The coexistence of propagating and quasilocalized states (P-states and Q-states) at the same energy is characteristic for QPCs and gives rise to indirect backscattering in the QPC.

The correction to the current due to backscattering can be written in a standard way [3, 13]:

$$
\begin{array}{r}
\Delta I=-\frac{2|e|}{h} \int d E\left(n_{F}\left(E-\mu_{1}\right)-n_{F}\left(E-\mu_{2}\right)\right) J(E) ; \\
J(E)=\sum_{m=1}^{N} \sum_{n=1}^{N}\left|r_{n m}(E)\right|^{2} .
\end{array}
$$

In the linear response limit this yields the correction to the conductance,

$$
\Delta G=-\frac{2 e^{2}}{h} J\left(E_{F}\right)
$$

The summation in (7) is taken only over $N$ propagating (open) modes in the quantum point contact, and $r_{n m}(E)$ is the probability amplitude of the electron incident from the right in $m$ th $\mathrm{P}$-mode $(\alpha=1)$ to be scattered back, to the $n$th $\mathrm{P}$-mode with $(\alpha=-1)$ :

$$
r_{n m}(E) \delta\left(E-E^{\prime}\right)=\left(\psi_{n,-1}\left(E^{\prime}\right), \Psi_{m, 1}^{o u t}(E)\right)
$$

Here $\Psi_{m, 1}^{o u t}(x, y ; E)$ is the scattered wave, corresponding to the unit wave with energy $E$, incident from the right in the $m$ th mode, $\psi_{m, 1}(x, y ; E)$. This function can be found from the Lippmann-Schwinger equation [14] $(\mathbf{r}=(x, y))$ :

$$
\Psi_{m, 1}^{\text {out }}(\mathbf{r} ; E)=\psi_{m, 1}(\mathbf{r} ; E)+\int d \mathbf{r}^{\prime} G^{R}\left(\mathbf{r}, \mathbf{r}^{\prime} ; E\right) V\left(\mathbf{r}^{\prime}\right) \psi_{m, 1}\left(\mathbf{r}^{\prime} ; E\right)
$$

In this equation we have introduced the exact retarded Green's function (retarded Green's function) in the presence of the perturbation $V(x, y)$, for which we can write :

$$
G^{R}\left(\mathbf{r}, \mathbf{r}^{\prime} ; E\right)=G_{0}^{R}\left(\mathbf{r}, \mathbf{r}^{\prime} ; E\right)+\int d \mathbf{r}^{\prime \prime} G_{0}^{R}\left(\mathbf{r}, \mathbf{r}^{\prime \prime} ; E\right) V\left(\mathbf{r}^{\prime \prime}\right) G_{0}^{R}\left(\mathbf{r}, \mathbf{r}^{\prime} ; E\right)+\ldots
$$


The unperturbed retarded Green's function is given by

$$
G_{0}^{R}\left(\mathbf{r}, \mathbf{r}^{\prime} ; E\right)=\sum_{m, \alpha, \epsilon} \frac{\psi_{m, \alpha}(\mathbf{r} ; \epsilon) \psi_{m, \alpha}^{*}\left(\mathbf{r}^{\prime} ; \epsilon\right)}{E-\epsilon+i 0} \equiv \sum_{\alpha} G_{0}^{R ; \alpha}\left(\mathbf{r}, \mathbf{r}^{\prime} ; E\right)
$$

The summation is taken over all the quantum numbers of electronic unperturbed eigenfunctions.

Inserting (10,11) into (9) we find two first nonvanishing terms:

$r_{n m}(E) \delta\left(E-E^{\prime}\right)=\left(\psi_{n,-1}\left(E^{\prime}\right), G_{0}^{R}(E) V \psi_{m, 1}(E)\right)+\left(\psi_{n,-1}\left(E^{\prime}\right), G_{0}^{R}(E) V G_{0}^{R}(E) V \psi_{m, 1}(E)\right)$.

We neglect the higher-order terms which is equivalent to an assumption that the scattering processes by different quasilocalized levels are independent. This assumption is valid only in the case $L \ll l_{t r}, l_{l o c}$, where $l_{t r}, l_{l o c}$ are the transport and localization lengths, respectively. The following results show that this condition holds in the case of realistic quantum contacts.

According to our initial assumptions, the perturbation potential is soft. This means that, generally, its matrix elements between the P-states propagating in opposite directions (i.e., with $\alpha=+1$ and -1 ) are negligible, while the matrix elements between the P-states and Q-states $(\alpha= \pm 1$ and 0$)$ are nonzero. Since the retarded Green's function (12) contains the products of electronic eigenfunctions with the same indices $\alpha$, Eq.(13) reduces to:

$$
r_{n m}(E) \delta\left(E-E^{\prime}\right)=\left(\psi_{n,-1}\left(E^{\prime}\right), G_{0}^{R ;-1}(E) V G_{0}^{R ; 0}(E) V \psi_{m, 1}(E)\right)
$$

The superscripts in the retarded Green's functions show which part of the expansion (12) we keep.

The formula (14) shows that the backscattering from the incident $(\mathrm{m}, 1)$-state to the $(\mathrm{n},-1)$-state occurs through the set of quasilocalized states (described by the part of retarded Green's function, denoted by $\left.G_{0}^{R ; 0}(E)\right)$.

After some standard transformations, we obtain that

$$
r_{n m}(E)=2 \pi \nu(E) \sum_{q, \epsilon} \frac{(n ;-1 ; E|V| q ; 0 ; \epsilon)(q ; 0 ; \epsilon|V| m ; 1 ; E)}{E-\epsilon+i 0} .
$$


Here we have introduced the one-dimensional electronic density of states (DOS) at infinity, $\nu(E)$, to account for the propagating modes, and denoted the matrix elements of the perturbation potential by

$$
(n ;-1 ; E|V| q ; 0 ; \epsilon) \equiv \int d \mathbf{r} \psi_{n,-1}^{*}(\mathbf{r} ; E) V(\mathbf{r}) \psi_{q, 0}(\mathbf{r} ; \epsilon)
$$

The summation is now taken only over the quasilocalized states; as such, they have discrete spectrum, so that $\epsilon$ is a discrete variable; $q$ is the number of transverse mode where the localized state appears.

The equation (15) contains the unperturbed retarded Green's function of the electron in localized states, which thus have an infinite lifetime. The perturbation, mixing different states, makes the Q-states metastable, and instead of $i 0$-term there appears the spectral function $i \Gamma_{q}(\epsilon, \epsilon) / 2$ [14] (the accompanying shift of the energy levels can be accounted for by changing the summation variable $\epsilon$ ). The spectral function is given by

$$
\Gamma_{q}\left(\epsilon, \epsilon^{\prime}\right)=2 \pi \sum_{n} \sum_{\alpha= \pm 1}\left|\left(q ; 0 ; \epsilon|V| n ; \alpha ; \epsilon^{\prime}\right)\right|^{2} \nu(\epsilon)
$$

(of course, a Q-state can decay only into P-states).

The correction to the current (6) is expressed through $J(E)=\sum_{m, n}\left|r_{n m}(E)\right|^{2}$. Note that the matrix elements corresponding to the Q-states from different subbands $\left(q \neq q^{\prime}\right)$ or different impurity potential wells in the same subband $\left(w \neq w^{\prime}\right.$, where $w$ is the label of the potential well) enter the expression for $r_{n m}(E)$ with their phases, which are uncorrelated. This means that the main contribution to $J(E)$ will be given by the diagonal terms in the corresponding sum, that is, as it is easily seen,

$$
\sum_{q, w} \sum_{\epsilon, \epsilon^{\prime}} \frac{\sum_{m, n}(n ;-1 ; E|V| q ; 0 ; \epsilon)(q ; 0 ; \epsilon|V| m ; 1 ; E) \cdot\left(n ;-1 ; E|V| q ; 0 ; \epsilon^{\prime}\right)\left(q ; 0 ; \epsilon^{\prime}|V| m ; 1 ; E\right)}{\left(E-\epsilon+i \Gamma_{q}(\epsilon, \epsilon) / 2\right)\left(E-\epsilon^{\prime}-i \Gamma_{q}\left(\epsilon^{\prime}, \epsilon^{\prime}\right) / 2\right)} .
$$

Here $\epsilon, \epsilon^{\prime}$ are different quasilocalized states in the same potential well of the same 1D subband.

This expression can be further simplified, if the distance between the energy levels in a potential well are large comparatively to their width: $\Delta \epsilon_{\text {well }} \gg \Gamma$. Then in the sum over $\epsilon, \epsilon^{\prime}$ in $(18)$ the terms with $\epsilon \neq \epsilon^{\prime}$ are negligibly small, so that finally we obtain: 


$$
\begin{array}{r}
J(E) \simeq 4 \pi^{2} \nu^{2}(E) \sum_{q, \epsilon} \frac{\sum_{m, n}|(n ;-1 ; E|V| q ; 0 ; \epsilon)|^{2}|(q ; 0 ; \epsilon|V| m ; 1 ; E)|^{2}}{(E-\epsilon)^{2}+\left(\Gamma_{q}(\epsilon, \epsilon) / 2\right)^{2}} \\
=\sum_{q, \epsilon} \frac{\left(\Gamma_{q}(\epsilon, E) / 2\right)^{2}}{(E-\epsilon)^{2}+\left(\Gamma_{q}(\epsilon, \epsilon) / 2\right)^{2}} .
\end{array}
$$

The result has a typical Breit-Wigner form for a set of independent resonant levels [14], which is the case under the assumptions made.

Though the energy spectrum of the Q-states in a single potential well is assumed to be rarified, in a long contact, where many potential wells with random parameters appear, and due to the fact that each Q-state has a finite width $\Gamma$, the spectrum of these states for the whole system is dense enough to be described in countinuous approximation. Then we can introduce the density of localized states in the $q$ th mode, $\aleph_{q}(\epsilon)$, and rewrite (19) as

$$
J(E)=\sum_{q} \int d \epsilon \frac{\aleph_{q}(\epsilon)\left(\Gamma_{q}(\epsilon, E) / 2\right)^{2}}{(E-\epsilon)^{2}+\left(\Gamma_{q}(\epsilon, \epsilon) / 2\right)^{2}} \simeq \frac{\pi}{2} \sum_{q} \aleph_{q}(E) \Gamma_{q}(E, E) .
$$

In the linear response limit this directly gives the correction to the conductance:

$$
\Delta G \simeq-\frac{2 e^{2}}{h} \frac{\pi}{2} \sum_{q} \aleph_{q}\left(E_{F}\right) \Gamma_{q}\left(E_{F}, E_{F}\right) .
$$

We discussed only virtual (including resonant) scattering to and from the Q-states. In reality, when the finite driving voltage $U$ is applied, there exist the processes of real elastic and inelastic (electron-electron or electron-phonon) scattering between Q- and P-states with energies in the interval $e U$ around the Fermi energy. The contribution of these states to the scattering is proportional to $e U$. We can safely neglect their existence in the if

the linear response limit $e U \rightarrow 0$, when the contribution from the Q-states outside the $e U$-band (in the interval of width $\sim \Gamma\left(E_{F}, E_{F}\right)$ ) is dominant. These kinetic processes, of course, must be taken into account if we would like to discuss the nonlinear response of a QPC.

\section{STATISTICS OF CONDUCTANCE FLUCTUATIONS}

Now let us discuss what the formula (21) yields. The conductance quantization breakdown can be characterized by the relative difference between the actual conductance 
vs. gate voltage dependence, $G\left(V_{g}\right)$, and the ideal one, averaged over the $n$-th step (Fig.],inset) (reduced conductance deviation):

$$
g_{n}=\left(2 e^{2} / h\right)^{-1} \frac{\int_{V_{g}(n)}^{V_{g}(n+1)} d V_{g}\left|\Delta G\left(V_{g}\right)\right|}{V_{g}(n+1)-V_{g}(n)} .
$$

Inserting here (21), we get:

$$
g_{n}=\frac{\pi}{2} \sum_{q} \int_{V_{g}(n)}^{V_{g}(n+1)} d V_{g} \aleph_{q}\left(E_{F}\right) \frac{\Gamma_{q}\left(E_{F}, E_{F}\right)}{V_{g}(n+1)-V_{g}(n)},
$$

where the $n$-th mode opens at the gate voltage equal to $V_{g}(n)$. In the last equation we can instead of integrating over the gate voltage integrate over the subband bottom energies, $E_{\perp}(x)$, which are determined by $V_{g}$. The intermode distance, $\Delta E_{\perp}(x)$, weakly depends on the gate voltage, the main effect of which was directly shown to be an upward shift of the potential in the constriction [17]. In the realistic model of a parabolic confining potential it also does not depend on the mode number, and in the adiabatic limit is almost coordinate independent. Its magnitude is of the order of $E_{F} / N_{\max }$, where $N_{\max }$ is the number of transverse modes in the wide part of the constriction (and equals approximately to the number of conductance $2 e^{2} / h$-steps which can be observed in given QPC). This enables us to simplify the above equation:

$$
g_{n} \approx \frac{\pi}{2} \sum_{q} \int_{E_{q \perp}}^{E_{q \perp}+\Delta E_{\perp}} d E_{\perp} \aleph_{q}\left(E_{F}\right) \frac{\Gamma_{q}\left(E_{F}, E_{F}\right)}{\Delta E_{\perp}} .
$$

The functions under the integral are implicit functions of the bottom position of the corresponding mode.

If localized states have an almost continuous spectrum (which is consistent with our initial assumption of the impurity potential being smooth), the main dependence on the bottom energy of the mode enters the DOS, not the level width, which is a smooth function of energy in the scale of $\Delta E_{\perp}$. Therefore we can take it from under the integration. Then we obtain the following formula:

$$
g_{n} \approx \frac{\pi}{2} \cdot \frac{\Gamma\left(E_{F}, E_{F} ; n\right)}{\Delta E_{\perp}} \int_{E_{q \perp}}^{E_{q \perp}+\Delta E_{\perp}} d E_{\perp} \sum_{q} \aleph_{q}\left(E_{F}\right) .
$$

Here we explicitly show that, as follows from the definition (17),$\Gamma_{q}\left(E_{F}, E_{F}\right)$ depends on the number of propagating states in the contact, that is, on the step number $n$. 
The integral over energy in (25) has a sense of number of localized states in all 1D subbands in the contact, which pass the Fermi level as we sweep $V_{g}$ across the $n$-th conductance step, $N_{Q}$. We will denote its average over possible realizations of the random potential by $\kappa L(L$ is the length of the contact):

$$
\kappa=\frac{\left\langle N_{Q}\right\rangle}{L}=\frac{1}{L} \int_{E_{q \perp}}^{E_{q \perp}+\Delta E_{\perp}} d E_{\perp}\left\langle\sum_{q} \aleph_{q}\left(E_{F}\right)\right\rangle,
$$

or

$$
\kappa=\int_{E_{q \perp}}^{E_{q \perp}+\Delta E_{\perp}} d E_{\perp}\left\langle\sum_{q} \mathcal{N}_{q}\left(E_{F}\right)\right\rangle .
$$

Here $\left\langle\mathcal{N}_{q}(E)\right\rangle$ is the ensemble average of DOS per unit length in the $q$-th 1D subband over possible realizations of the random impurity potential, so that the ensemble average of $g_{n}$ is

$$
\left\langle g_{n}\right\rangle=\frac{\pi}{2} \cdot \frac{\left\langle\Gamma\left(E_{F}, E_{F} ; n\right)\right\rangle}{\Delta E_{\perp}} \kappa L .
$$

Now the expression (25) acquires a clear physical meaning. The correction to the contact conductance due to indirect backscattering is a sum of contributions from independent Q-states (in each 1D subband):

$$
g_{n}=N_{Q} \Delta g_{n}
$$

Each contribution is approximately the same and is proportional to $n$ (see (17)) :

$$
\Delta g_{n}=\frac{\pi\left\langle\Gamma\left(E_{F}, E_{F} ; n\right)\right\rangle}{2 \Delta E_{\perp}}=\gamma n .
$$

The density of states is the self-averaging quantity [15], so that in the limit of infinitely long quantum contact we would get :

$$
\begin{array}{r}
\lim _{L \rightarrow \infty} \frac{\aleph_{q}(E)}{L}=\left\langle\mathcal{N}_{q}(E)\right\rangle, \\
\Rightarrow \lim _{L \rightarrow \infty} \frac{N_{Q}}{L}=\kappa .
\end{array}
$$

In the contact of finite length the number of appropriate Q-states deviates from its ensemble average, $\left\langle N_{Q}\right\rangle=\kappa L$, and thus the conductance of the QPC fluctuates from 
realization to realization. In order to predict the performance of a single QPC, we need the distribution function of these fluctuations, dependent on such parameters of the contact as its length $L$ and number of open modes $n$.

On a length scale large compared to the size of the localized state (correlation radius of the impurity potential, of order $100 \mathrm{~nm}$ ), we can regard these states as independently randomly distributed along the channel, with occurence per unit length $\kappa$. The probability to find $p$ such states along the total length $L$ of the contact is then given by the Poisson formula [18]:

$$
\Pi(N)=(\kappa L)^{N} e^{-\kappa L} / N !
$$

Since each state gives the same contribution to the conductance, $\Delta g_{n}=\gamma n$, then the probability density of the conductance deviation on the $n$-th step

$$
P\left(g_{n}=N \gamma n\right)=\frac{(\kappa L)^{g / \gamma n} e^{-\kappa L}}{\gamma n(g / \gamma n) !} .
$$

The reduced conductance deviation acquires the discrete set of values, $g_{n}=$ $\gamma n, 2 \gamma n, 3 \gamma n \ldots$, depending on the number of relevant Q-states in the contact.

The shortcoming of the above formula (discrete set of values of $g_{n}$ ) follows from our simplifying assumption that each Q-state has the same width $\left\langle\Gamma\left(E_{F}, E_{F}\right)\right\rangle$. In reality, this quantity also fluctuates, so that the variable $g_{n}$ is rather a continuous one. Nevertheless, as the numerical calculations show, this expression provides a fairly good description of the performance of a QPC (see Sec.VI).

\section{ESTIMATES OF THE THEORY PARAMETERS}

We see that the statistics of the conductance fluctuations due to indirect backscattering is determined by the following parameters (see Eq.(34)): effective length of the QPC, $L$, number of open modes, $n$, average 1D density of Q-states at the Fermi energy per unit length, $\kappa$, and average contribution to the conductance from each Q-state, $\Delta g$. Since

the latter quantity is proportional to the average scattering rate from these states to the propagating ones (see (17)), 


$$
\Delta g=\frac{\pi}{2} \frac{\left\langle\Gamma\left(E_{F}, E_{F} ; n\right)\right\rangle}{\Delta E_{\perp}}=\gamma n,
$$

it is more convenient to use the set of four independent parameters, $L, n, \kappa$ and $\gamma$.

The first two are tunable parameters, which can be changed by changing $V_{g}$ and/or gate configuration, while the other two are essentially determined by the properties of given GaAs structure (density and charge of impurities, spacer thickness etc.), or, in short, by the impurity potential in the system. We need some numerical estimates for these intrinsic parameters, $\gamma$ and $\kappa$. They can be obtained from such characteristics of the impurity potential as its correlation length, $l_{V}$, and dispersion, $\sigma^{2} \equiv\left\langle V_{i m p}^{2}\right\rangle$, which are both contained in the correlation function $K(\mathbf{x})$ or spatial spectral density $S(\mathbf{k})$ (see [16]):

$$
K(\mathbf{x})=\left\langle V_{i m p}(0) V_{i m p}(\mathbf{x})\right\rangle ; \quad S(\mathbf{k})=\int d^{d} \mathbf{x} K(\mathbf{x}) e^{-i \mathbf{k} \cdot \mathbf{x}}
$$

(We put to zero the average value of the impurity potential.)

First we estimate $\gamma$. Since $\left\langle\Gamma\left(E_{F}, E_{F}\right)\right\rangle \simeq 2 \pi \cdot n \cdot 2 \nu \overline{|(Q|V| P)|^{2}}$ (see (17)), then

$$
\gamma \simeq 2 \pi^{2} \nu \overline{|(Q|V| P)|^{2}} / \Delta E_{\perp}
$$

Here $\overline{|(Q|V| P)|^{2}}$ is the average square modulus of the impurity potential between quasilocalized and propagating states. Evidently, it is of the order of $S\left(\Delta k_{P Q}\right) \alpha^{2} / l_{0}$, where $\Delta k_{P Q}$ is the difference of longitudinal wave vectors in $\mathrm{P}$ - and $\mathrm{Q}$ - states, $\alpha$ is the reduced matrix element of impurity potential between different transverse modes $(\alpha \simeq 0.1$ according to our numerical calculations, see Sec.(V), and $l_{0} \simeq l_{V}$ is the average longitudinal size of the quasilocalized state (the latter factor appears because the wave functions of Q-states are normalized to unit probability, while the P-states are normalized to unit probability current along the axis of the QPC). In accordance with our basic assumptions, for the relevant (Q,P)-matrix elements of the impurity potential the longitudinal wavevectors are almost the same: $\Delta k_{P Q} \ll k_{F}$. Therefore we can take

$$
\overline{|(Q|V| P)|^{2}} \simeq S(0) e^{-2 \Delta k_{P Q} l_{V}} \alpha^{2} / l_{V}=S(0) e^{-4 \pi \frac{l_{V}}{\lambda_{F}} \frac{\Delta k_{P Q}}{k_{F}}} \alpha^{2} / l_{V} \simeq \sigma^{2} l_{V} \alpha^{2}
$$

This gives the following estimate for $\gamma$ : 


$$
\gamma \leq 2 \pi^{2} \alpha^{2} \frac{\sigma^{2}}{E_{F} \Delta E_{\perp}} \cdot \frac{l_{V}}{\lambda_{F}} .
$$

We use here the formula for 1D DOS of propagating states, $\nu=1 / \pi \hbar v_{F}$.

If we insert into (39) the values consistent with our numerical calculations, $\alpha=0.1$, $\lambda_{F}=40 \mathrm{~nm}, l_{V}=100 \mathrm{~nm}, \sigma^{2}=0.1 E_{F}^{2}, \Delta E_{\perp}=0.3 E_{F}$, then we obtain $\gamma \leq 0.15$. This is a proper order of magnitude estimate, though higher than the value that follows from the numerical calculations (see Sec.VI). The reason is, that putting $\Delta k_{P Q}=0$ in (38) we overestimated $\gamma$. To eliminate this discrepancy, it is sufficient to take $\Delta k_{P Q} / k_{F} \simeq 7 \%$, which is quite reasonable.

One useful remark: In the superposition approximation (see, e.g., [16]) we can present the impurity potential as a sum of potentials of identical potential centres, $v(x)$, with density $n_{i m p}$ randomly distributed along the conducting plane. Then by the Carson theorem we get 18$] S(k)=n_{\text {imp }}|v(k)|^{2}$. Therefore the parameter $\gamma$ is proportional to the impurity density.

In order to estimate the other intrinsic parameter, $\kappa$, we use the quasiclassical, or Thomas-Fermi, approximation for the average density of localized states in the 1D subband, $\langle\mathcal{N}(E)\rangle$ (see 16]), leading to the following approximate expression:

$$
\begin{aligned}
\left\langle\mathcal{N}_{q}(E, x)\right\rangle=\frac{\sqrt{m}}{\pi \hbar \sqrt{\sigma}} \theta\left(E_{q \perp}(x)-E\right) & \exp \left(-\frac{\left(E_{q \perp}(x)-E\right)^{2}}{4 \sigma^{2}}\right) \mathrm{D}_{-1 / 2}\left(\frac{E_{q \perp}(x)-E}{\sigma}\right) \approx \\
& \approx \frac{\theta\left(E_{q \perp}(x)-E\right)}{\sqrt{E_{q \perp}(x)-E}} \frac{\sqrt{m}}{\pi \hbar} \exp \left(-\frac{\left(E_{q \perp}(x)-E\right)^{2}}{2 \sigma^{2}}\right) .
\end{aligned}
$$

$\mathrm{D}_{-1 / 2}(z)$ is the parabolic cylinder function. As a matter of fact, the validity limits of the latter expression are determined not by the quasiclassicity conditions, but by much looser ones [15]:

$$
\left|E-E_{q \perp}(x)\right| \gg \sigma, E_{F} \cdot \frac{\lambda_{F}^{2}}{l_{V}^{2}} .
$$

Substituting it into the definition of $\kappa$ (27), we find:

$$
\begin{aligned}
& \kappa \approx \sum_{q} \int_{E_{q \perp}}^{E_{q \perp}+\Delta E_{\perp}} d E \frac{\theta\left(E-E_{F}\right)}{\sqrt{E-E_{F}}} \frac{\sqrt{m}}{\pi \hbar} e^{-\frac{\left(E-E_{F}\right)^{2}}{2 \sigma^{2}}} \\
& \approx \int_{E_{F}+\Delta E_{\perp}}^{\infty} \frac{d E}{\Delta E_{\perp}} \Delta E_{\perp} \frac{1}{\sqrt{E-E_{F}}} \frac{\sqrt{m}}{\pi \hbar} e^{-\frac{\left(E-E_{F}\right)^{2}}{2 \sigma^{2}}} .
\end{aligned}
$$


The latter formula can be identically rewritten as follows:

$$
\kappa \approx 2^{-1 / 4} \boldsymbol{\Gamma}\left(\frac{1}{4}, \frac{1}{2}\left(\frac{\Delta E_{\perp}}{\sigma}\right)^{2}\right) \cdot \sqrt{\frac{\sigma}{E_{F}}} \cdot \frac{1}{\lambda_{F}} .
$$

Here $\boldsymbol{\Gamma}(\alpha, z)=\int_{z}^{\infty} d y y^{1-\alpha} e^{-y}$ is the incomplete gamma function.

With the same choice of parameter values as above, we find that $\kappa \simeq \sqrt{0.66}$ $\Gamma(1 / 4,1 / 2) / \lambda_{F}=0.269 / \lambda_{F} \approx 0.006 \mathrm{~nm}^{-1}$.

\section{NUMERICAL CALCULATIONS}

The basis for our hypothesis about the statistics of conductance deviations from ideal quantization is the numerical calculations of QPC conductance for different realizations of soft random impurity potential, at different contact lengths and for different number of open modes.

The model used for simulation of a heterostructure device is very similar to the one considered in [4]. Donors are assumed to be fully ionized and distributed randomly through the donor layer. We restricted donors to a plane which should be considered as the middle of the donor layer. In our calculations all the donors at the $n$ - type $\mathrm{Al}_{x} \mathrm{Ga}_{1-x} \mathrm{As}_{\text {- }}$ doped layer have the same height $h=30 \mathrm{~nm}$ above the 2DEG. The electrons are treated as a twodimensional layer of $10 \mathrm{~nm}$ thickness, which is much smaller than other relevant length scales. The positions of the impurities were generated by the uniform random number generator at the square $1290 \times 1290 \mathrm{~nm}^{2}$. We assumed periodic boundary conditions, i.e. this square was continued periodically in all directions in $2 \mathrm{D}$ plane. In order to avoid the occasional appearance of too dense clusters of impurities at the donor plane the distribution of impurities has been 'relaxed', namely, we did not allow the appearance of more than 5 impurity atoms at the area $1 / n$, where $n$ is the $2 \mathrm{D}$ concentration of impurities. For the realistic concentration of impurities, $n=10^{12} \mathrm{~cm}^{-2}$ one must discard not more than $5 \div 10 \%$ of impurities. The potential of a single impurity was taken in the following form:

$$
v\left(\mathbf{r}-\mathbf{r}_{i}\right)=\frac{e}{\varepsilon \sqrt{\left(\mathbf{r}-\mathbf{r}_{i}\right)^{2}+h^{2}}},
$$


where $\varepsilon(\approx 13)$ is the static dielectric function of GaAs, $\mathbf{r}$ is the position vector in the $2 \mathrm{D}$ plane, and $\mathbf{r}_{i}$ is the coordinate of i-th impurity. Since we did not want to be too specific in modelling any definite heterostructure, we did not take into account the image term considered in [4].

The potentials of the impurities were summed directly. The numerical calculations show that the summation of the potentials of impurities situated within the radius $\mid \mathbf{r}-$ $\mathbf{r}_{i} \mid \leq r_{\max }=10 h$ gives the resulting potential with a good precision. For the rest of the plane the summation could be substituted by integration over a plane with homogeneously distributed charge. This gives a constant term, which can be dropped since we are only interested in the fluctuations of the impurity potential around its average value. Therefore, we choose the average value of the summed potential as a point of reference for the energy, i.e. we take $\left\langle V_{i m p}\right\rangle=0$. Further increase of $r_{\max }$ does not give any significant change for the potential fluctuations defined in such a way.

The amplitude of fluctuations of the unscreened impurity potential proves to be too large, for a realistic concentration of impurities $n \sim 10^{12} \mathrm{~cm}^{-2}$ being a few times greater than the Fermi energy (of the order of $10^{-2} \mathrm{eV}$ ). This means that the screening of this potential by the electrons in 2DEG should necessarily be taken into account. For a qualitative estimation of this effect we used the way proposed in [四]: the Thomas-Fermi approximation, which is applicable in the case of slowly varying (on the scale of $\lambda_{F}$ ) impurity potential, i.e., our case. The density of electrons is then given by the local equation

$$
n(\mathbf{r})=\frac{m^{*}}{\pi \hbar^{2}}\left(\mu-e V_{t o t}(\mathbf{r})\right) \theta\left(\mu-e V_{t o t}(\mathbf{r})\right),
$$

where $\mu$ is the chemical potential, and $V_{\text {tot }}$ is a sum of the unscreened impurity potential and the induced potential from the electrons in 2DEG,

$$
V_{\text {tot }}=V_{i m p}+V_{i n d}
$$

$V_{\text {ind }}$ is related to $n_{\text {ind }}$ by the Poisson equation. For a fixed chemical potential Eqs. (45) and (46) give a possibility to find the total potential $V_{\text {tot }}$ in a self-consistent manner. One 
must keep in mind that the experimentally observed Fermi energy should be measured from the average total potential, namely, one more equation should be added to this self-consistent scheme,

$$
E_{F}=\mu-\left\langle V_{t o t}\right\rangle
$$

Here $\left\langle V_{t o t}\right\rangle$ is the average total potential, $E_{F}$ is considered as a fixed parameter given by the experiment, $\mu$ is the "bare" chemical potential. It shows the degree of filling of the system by electrons and varies from iteration to iteration. Obviously, this system of equations has a single self-consistent solution. The numerical calculations show that for $n \sim 10^{12} \mathrm{~cm}^{-2}$ and $E_{F} \simeq 10^{-2} \mathrm{eV}$ this solution is achieved when the system is almost completely filled by the electrons, i.e., for the most of the points in $2 \mathrm{D}$ plane $n_{\text {ind }}$ is non-zero and the resulting potential fluctuations are smaller than (or of the order of) the Fermi energy, $V_{t o t}-\left\langle V_{t o t}\right\rangle \leq E_{F}$ (see Fig.1.) The characteristic scale of the change of the potential fluctuation is of the order of $100 \div 200 \mathrm{~nm}$.

The calculations of the potential were made with a crude mesh in $2 \mathrm{D}$ plane. To solve the scattering problem by the standard transfer matrix method, a finer mesh is desirable, and the potential was therefore interpolated by the cubic splines when necessary.

In all these considerations we did not take into account the self-consistent effects of redistribution of electrons in the constriction due to the gate voltage. Nevertheless, it is clear that such effects could be very significant, since it could enhance the potential of a single impurity located in a bottle neck because the concentration of electrons in this area is lower than the average one, and screening is suppressed (impurity denudation effect). Such an effect increases the potential fluctuations at the bottle neck and can change significantly the whole behaviour of the conductance (locking the channel) [4]. The question of how this effect of just a few impurities located at the narrowest place may show up in the statistics of conductance fluctuations is still open. We plan to discuss such a challenging subject elsewhere.

In order to obtain the transmittance and reflectance matrices we solved the $2 \mathrm{D}$ Schrödinger equation rewriting it in matrix form by the standard transfer matrix method 
with the proper boundary conditions. In order to model the gate voltage we used a parabolic potential, as in [6],

$$
U_{g}(x, y)=\hbar^{2} \eta^{2} / 2 m^{*}[2 y / b(x)]^{2},
$$

where $\eta=1 / 4 k_{F} b(x)$, and

$$
b(x)=b_{\infty}-\left(b_{\infty}-b_{0}\right) \sin ^{2}(\pi x / L) ; \quad-L / 2 \leq x \leq L / 2 .
$$

We chose the parameters $b_{0}=10 \mathrm{~nm}$ and $b_{\infty}=170 \mathrm{~nm}$, i.e., the maximum number of the modes passing through the constriction is eight, for the realistic $\lambda_{F}=42 \mathrm{~nm}$.

As long as we are interested mostly in long constrictions (of length $600 \mathrm{~nm}$ and longer) this impurity scattering term will be dominating in comparison with effects of changes of the width $b(x)$ along the constriction, a case thoroughly considered by Brataas and Chao [6] for short contacts. In the bulk of our calculations we neglect them. Because the most important region for the breakdown of quantization lies near the bottleneck, we multiply the impurity potential inside the contact by the factor $\sin ^{2}(\pi x / L)$. This gives us the freedom not to care about changing the boundary conditions for any realization of the potential. The number of entering modes is always constant.

The statistics of conductance deviations in quantum contacts can be obtained either (i) by generating the impurity potential (with the self-consistent procedure) for each realization of the constriction, or (ii) by moving the center of the contact (possibly also changing the direction of the axis of the contact) over the plane with the impurity potential obtained in a self-consistent manner only once. The second way is surely less time consuming and, therefore, is the one we use.

We performed numerical calculations for contacts of three lengths (600, 800 and 1200 $\mathrm{nm}$ ) for 625 different realizations of impurity potential, moving the center of the X-oriented contact along the square of $1290 \times 1290 \mathrm{~nm}^{2}$, with the periodic boundary conditions described above.

We also calculated the conductance of very long QPCs (up to $10000 \mathrm{~nm}$ ) for several realizations. 


\section{ANALYSIS OF THE RESULTS}

The results of the calculations are shown in Figs.3, 4. 4 and 5. Typical conductance vs. gate voltage curves are shown in Fig. 3. Curves A correspond to an impurity free contact. The center of the contact lies at the same place for all the curves B (at a minimum (valley) of the impurity potential) and $\mathrm{C}$ (at a maximum (hill)). Evidently the C-curves demonstrate better quantization than the B-curves, for the same contact length and step number. This agrees with our statement that the indirect backscattering is more effective mechanism of the quantization breakdown in QPCs. The quantization quality becomes poorer for larger contact length and higher step number.

The conductance vs. gate voltage curves in a very long QPC $(\mathrm{L}=10000 \mathrm{~nm})$ for three different realizations are shown in Fig. 1 . We would like to note here that no trace of localization in the system is detected. On the other hand, the conductance plateaus (except the first one) are totally destroyed at this length.

In order to obtain more convincing proof of our theory, the statistics of conductance fluctuations was investigated. The average conductance deviation from the ideal conductance step , $g$, was calculated in 625 realizations for each step $(n=2,3, . ., 6)$ and each contact length $(L=600,800,1200 \mathrm{~nm})$, and the empirical distribution function was obtained:

$$
P_{e m p}(g) \delta g=N(p \delta g) / 625
$$

where $N(p \delta g)$ is the number of realizations for which $p \delta g \leq g<(p+1) \delta g$. In the actual calculations $\delta g=0.02$.

Since the contact has a finite length, the deviation $g$ is finite even without impurities due to the geometric step smoothening [19]; it is the larger the shorter the contact is. Therefore when calculating $g$, we have substracted from it the corresponding value of $g$ for the impurity free case.

The average deviation increases with the mode number and the contact length. The empirical distributions are essentially asymmetric. Some of them have a tail stretching into the negative $g$ region. This means that in some cases the impurity potential 
can make the contact more transparent in comparison with the case without impurities. There may be two mechanisms for this transparency growth. First, there may occur tunneling through the localized states in the transition regime, which enhances the contact transparency [12]; second, the random impurity potential may effectively lessen the aperture of the contact, thus making the conductance steps sharper and enlarging $g$ [19]. Both mechanisms are consistent with the fact that the negative tails disappear for larger contact length and/or step number, and both processes are likely to occur in different random potential arrangements.

The values of $g$ obtained from the numerical calculations are not quantized, as the expression (34) implies. This is, as stated above, due to the fact that the widths of different quasilocalized states are not exactly the same. Nevertheless, by interpolating this formula to noninteger values of $g$ we achieve a strikingly good description of the numerical results:

$$
P_{n}(g)=\frac{(\kappa L)^{g / \gamma n} e^{-\kappa L}}{\gamma n \boldsymbol{\Gamma}((g / \gamma n)+1)}
$$

This result can be easily understood, if we take into account that the contribution of each single Q-state to the correction to the conductance is small enough ( a few percent of the total effect, see below), so that a comparatively large number of the indirect backscattering processes is necessary to obtain the average effect in the QPC. Then the discrete distribution (34) almost coincides with its continuous interpolation (51). This is the more true, the longer the contact is. Indeed, the fitting of the two curves is better for the larger values of $L$ (see Fig.5).

The solid lines in Fig.5 are the least square fits of Eq.(51) to the empirical distribution. The dots on these lines indicate the points where $g$ is an integer multiple of $\gamma n$ (according to (34). We use the same value of the parameter $\kappa=(1 / 320) \mathrm{nm}^{-1}=0.13 / \lambda_{F}$ for all the curves, while the parameter $\gamma$ varied (the mean square error of the fitting was in the most cases practically the same as if we would vary them both independently). The choice of $\kappa$ agrees very well with the estimates of Sec IV $\left(\kappa \approx 0.269 / \lambda_{F} \approx 0.006 \mathrm{~nm}^{-1}\right)$, up to a factor of 2. This shows that the effective contact length where the scattering occurs is of 
the order of $L / 2$, i.e., coincides with the length of its bottleneck part (see Fig.回).

The values of $\gamma$ are shown in Table @. They change insignificantly with $L$ and $n$, and are in a good agreement with the estimates of Sec.ㅍ. The theoretical curves provide very good description of both position and magnitude of the distribution peak, as well as of its large deviation tail.

It is noteworthy that the width of the resonant peaks in $G\left(V_{g}\right)$ curves (Fig.3) agrees with our estimates of the resonance width $\Gamma \simeq \gamma n \Delta E_{\perp}\left(\Delta E_{\perp}\right.$ being of the order of the stepwidth).

The knowledge of the distribution function of conductance fluctuations allows to obtain more accurate criteria of good performance of a QPC, e.g., to predict the probability for a QPC of a given size, realized in the GaAs structure with given properties, to have a certain number of well-defined conductance steps.

The analysis of the theory parameters $\gamma$ and $\kappa$ shows that for different experimental situations they can be estimated as follows:

$$
\begin{array}{r}
\gamma \simeq 0.016 \cdot \frac{n_{\text {imp }}}{n_{0}} ; \\
\kappa\left(\mathrm{nm}^{-1}\right) \simeq \frac{1}{320} \cdot \exp \left(1-\frac{n_{0}}{n_{\text {imp }}}\left(\frac{\Delta E_{\perp}}{\Delta E_{\perp, 0}}\right)^{2}\right) .
\end{array}
$$

Here $n_{i m p}, \Delta E_{\perp}$ are the actual parameters of the system, and $n_{0}=10^{12} \mathrm{~cm}^{-2}, \Delta E_{\perp, 0}=4.1$ $\mathrm{meV}$ [20] are the model parameters in our calculations.

The simplest criterion of the QPC performance is given by average deviation (see (34))

$$
\left\langle g_{n}\right\rangle=\kappa \gamma n L \simeq 5 \cdot 10^{-5} n L(\mathrm{~nm})
$$

For the channel length $L=600(800,1200) \mathrm{nm}$ and for a criterion of total quantization destruction $\langle g\rangle=0.5$, this gives $n_{\max } \approx 17(13,8)$, respectively. The analysis of conductance curves shows that the quantization is really destroyed at lower values of $\langle g\rangle \simeq 0.2$, i.e. $n_{\max } \approx 7(5,3)$, which is close to what we see in Fig. 3 .

The transport and localization lengths can be estimated from (54) as follows:

$$
l_{t r} \approx(\kappa \gamma n)^{-1} ; \quad l_{l o c} \geq l_{t r} \cdot n
$$


This leads to the following results: for $n$ open modes $l_{t r} \approx 20000 / n(\mathrm{~nm}) ; l_{l o c} \geq 20000(\mathrm{~nm})$. These values are much larger than experimentally reasonable contact lengths; they justify our theoretical approach of Sec.[1].

In the recent experiment [21] the quantized conductance was observed in an InAs/AlSb ballistic constriction with channel length $1000 \mathrm{~nm}$. Up to eight conductance steps were detected. Koester et al. suggest that much better performance of their device compared to ones utilizing $\mathrm{GaAs} / \mathrm{Al}_{x} \mathrm{Ga}_{1-x}$ As heterostructures is due to higher ratio of interlevel energy spacing to the amplitude of the impurity potential fluctuations. This is in a complete agreement with our theory, since the density of quasilocalized states (and the parameter $\kappa$ of the theory, see Eq.(43)) exponentially drops with growing $\Delta E_{\perp} / \sigma$.

\section{CONCLUSION}

In conclusion, we have investigated the effects of electronic scattering by the soft impurity potential in quantum point contacts both with use of an analytical model and by direct numerical calculations.

We have shown that the decisive mechanism of conductance quantization breakdown is due to the indirect backscattering of carriers via quasilocalized states at the Fermi level. For the realistic contact lengths they can be described in terms of independent scatterers, though the electron propagation is coherent. This is due to the smoothness of the scattering potentials, leading to very large scattering and localization lengths.

The performance of the quantum contact is shown to be strictly dependent on the ratio of the intermode distance to the amplitude of the random impurity potential (the larger the better).

For the first time we have obtained analytical and empirical formulas both for the conductance deviation due to this process and for the probability distribution of these deviations in an ensemble of contacts with realistic potentials. The latter has proved to be a generalized Poisson distribution.

The parameters of the distribution obtained numerically agree quite well with ana- 
lytical calculations based on general assumptions, thus confirming their applicability to the case of quantum transport through the QPC in the presence of the random impurity potential.

\section{ACKNOWLEDGMENTS}

We are grateful to Mats Jonson and Ilya Krive for fruitful discussions. 


\section{REFERENCES}

[1] B.J. van Wees, H. van Houten, C.W.J. Beenakker, J.G. Williamson, L.P. Kouwenhoven, D. van der Marel, and C.T. Foxon. Phys.Rev.Lett. 60, 848 (1988); D.A. Wharam, M. Pepper, H. Ahmed, J.E.F. Frost, D.G. Hasko, D.C. Peacock, D.A. Ritchie, and G.A.C. Jones. J.Phys.C: Solid State Phys., 21, L209 (1988).

[2] S. Washburn and R.A. Webb, Repts. on Prog. in Physics 55, 1311 (1992).

[3] L.I. Glazman, G.B. Lesovik, D.E. Khmel'nitskii, and R.I. Shekhter, Pis'ma Zh. Eksp. Teor. Fiz. 48, 218 (1988) [JETP Lett. 48, 238 (1991)].

[4] J.A. Nixon and J.H. Davies, Phys. Rev. B 41, 7929 (1990); M.J. Laughton, J.R. Barker, J.A. Nixon, and J.H. Davies, Phys.Rev. B 44, 1150 (1991); J.A. Nixon, J.H. Davies, and H.U. Baranger, Phys.Rev. B 43, 12638 (1991).

[5] M. Büttiker, Phys.Rev. B 41, 7906 (1990).

[6] A. Brataas and K.A. Chao, Mod. Phys. Lett. B, 7, 1021 (1993).

[7] A.M. Zagoskin, R.I. Shekhter, to be published.

[8] Song He and S. Das Sarma, Phys.Rev. B 48, 4629 (1993).

[9] D.L. Maslov, C. Barnes, and G. Kirczenow, Phys.Rev. Letters 70, 1984 (1993).

[10] L.I. Glazman and M. Jonson, Phys.Rev. B 44, 3810 (1991).

[11] They are only quasilocalized, since their total energy can be equal or exceed the energy of propagating states; see below.

[12] S.A. Gurvitz and Y.B. Levinson, Phys. Rev. B 47, 10578 (1993).

[13] J. Imry, in Directions in Condensed Matter Physics, ed. by G.Grinstein and G.Mazenko; World Scientific Publ., Singapore, 1986, p.101.

[14] T.-Y. Wu and T. Omura, Quantum Theory of Scattering, Prentice-Hall Inc., NJ, 1962. 
[15] I.M. Lifshitz, S.A. Gredeskul, and L.A. Pastur, Introduction in the theory of disordered systems, NY: Wiley, 1988.

[16] J. Ziman, Models of Disorder, Cambridge University Press, 1979.

[17] N.K. Patel, J.T. Nicholls, L. Martín- Moreno, M. Pepper, J.E.F. Frost, D.A. Ritchie, and G.A.C. Jones, Phys. Rev. B 44, 13549 (1992).

[18] M.J. Buckingham, Noise in Electronic Devices and Systems, Ellis Horwood Ltd., New York- Brisbane- Chichester- Ontario, 1983.

[19] A.M.Zagoskin and I.O.Kulik, Sov. J. Low Temp. Phys. 16, 533 (1990); E.N.Bogachek, A.M.Zagoskin, and I.O.Kulik, Sov. J. Low Temp. Phys. 16, 796 (1990).

[20] In fact, the value of $\Delta E_{\perp}=4.1 \mathrm{meV}$ coincides with directly measured in [17] distance between the first and second transverse modes, which ranged from 4.0 to $4.6 \mathrm{meV}$ for different gate voltages.

[21] S. J. Koester, C. R. Bolognesi, E. L. Hu, H. Kroemer, and M. J. Rooks, Phys.Rev. B 49, 8514 (1994). 


\section{TABLES}

\begin{tabular}{|c|c|c|c|c|c|c|}
\hline $\mathrm{L}(\mathrm{nm}) \backslash \mathrm{n}$ & 2 & 3 & 4 & 5 & 6 & $\bar{\gamma}(L)$ \\
\hline 600 & 0.019 & 0.018 & 0.018 & 0.017 & 0.016 & 0.018 \\
\hline 800 & 0.016 & 0.017 & 0.017 & 0.016 & 0.015 & 0.016 \\
\hline 1200 & 0.014 & 0.014 & 0.014 & 0.013 & 0.012 & 0.014 \\
\hline $\bar{\gamma}(n)$ & 0.016 & 0.016 & 0.016 & 0.015 & 0.014 & $<\gamma>=0.016$ \\
\hline
\end{tabular}

TABLE I. Parameter $\gamma$ as determined by the least square fitting of Eq.(51) to the numerical data for different contact lengths $L$ and step numbers $n$. Parameter $\kappa$ is kept equal to $(1 / 320)$ $\mathrm{nm}^{-1}$ for all $L, n$. 


\section{FIGURES}

FIG. 1. The model of a quantum point contact. The impurity potential in 2DEG layer. Impurity density is $10^{12} \mathrm{~cm}^{-2}$. Isolines are drawn through $1.3 \times 10^{-3} \mathrm{eV}$. The white line shows the gate equipotential $U_{g}^{(0)}(x, y)=E_{F}$ (unperturbed contact shape) for $L=800 \mathrm{~nm}$.

FIG. 2. Classification of electronic states in a QPC. $E_{m \perp}(x)$ - effective potential in $m$-th mode (1D subband). $(\alpha=-2)$ - reflected states incident from the left; $(\alpha=-1)$ - propagating states incident from the left; $(\alpha=0)$ - quasilocalized states; $(\alpha=1)$ - propagating states incident from the right; $(\alpha=2)$ - reflected states incident from the right.

FIG. 3. Conductance vs. gate voltage curves for different realizations and contact lengths (see text). Parameter $Z=k_{F} b(0) / \pi$. a) - contact length $\left.1200 \mathrm{~nm}, \mathrm{~b}\right)-800 \mathrm{~nm}, \mathrm{c}$ ) - $600 \mathrm{~nm}$. Inset: Quantization breakdown parameter $g$; it equals to the ratio of the area of dashed part of the conductance step to its total area.

FIG. 4. Conductance vs. gate voltage curves for three different realizations for the contact length $\mathrm{L}=10000 \mathrm{~nm}$ (see text). The random potential has been generated on the rectangle $1290 \times 12900 \mathrm{~nm}^{2}$ for these calculations.

FIG. 5. Statistics of conductance fluctuations in QPCs (see text). a) - contact length 1200 nm, b) - $800 \mathrm{~nm}, \mathrm{c})-600 \mathrm{~nm}$. 